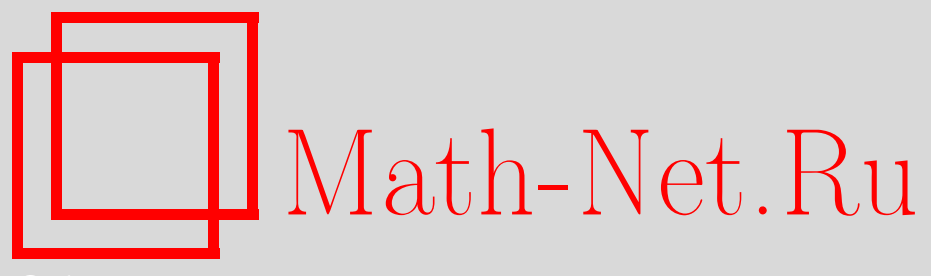

Ю. В. Малыхин, Усредненный модуль непрерывности и скобочная компактность, Матем. заметки, 2010, том 87, выпуск 3, 468-471

DOI: https://doi.org/10.4213/mzm8678

Использование Общероссийского математического портала Math-Net.Ru подразумевает, что вы прочитали и согласны с пользовательским соглашением http://www . mathnet.ru/rus/agreement

Параметры загрузки:

IP : 54.162 .127 .20

26 апреля 2023 г., 12:32:18

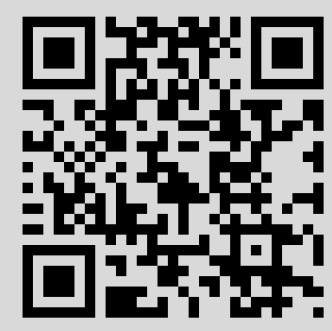




\section{Усредненный модуль непрерывности и скобочная компактность}

\section{Ю. В. Малыхин}

1. Определения. Пусть $f$ - ограниченная измеримая функция $\mathbb{T} \rightarrow \mathbb{R}$. Усредненным модулем непрерывности функции $f$ порядка $\delta>0$ называется величина

$$
\tau(f, \delta)=\int_{\mathbb{T}}\left(\sup _{|x-t| \leqslant \delta / 2} f(x)-\inf _{|x-t| \leqslant \delta / 2} f(x)\right) d t .
$$

Модуль $\tau$ был введен Сендовым и Поповым. В их книге [1] устанавливается связь $\tau$ с погрешностью односторонних приближений. В частности, ими доказывается, что величина наилучшего одностороннего приближения функции $f$ тригонометрическими полиномами степени не выше $n$ в метрике $L_{1}$

$$
E_{[]}\left(f, \mathscr{T}_{n}\right):=\inf \left\{\|P-Q\|_{1}: P(x) \leqslant f(x) \leqslant Q(x) \text { для всех } x \in \mathbb{T}, P, Q \in \mathscr{T}_{n}\right\}
$$

удовлетворяет аналогу неравенства Джексона:

$$
E_{[]}\left(f, \mathscr{T}_{n}\right) \leqslant 120 \cdot \tau\left(f, \frac{1}{n}\right) .
$$

Также отметим простое утверждение о том, что функция сколько угодно хорошо односторонне приближается непрерывными функциями в $L_{1}$, если и только если

$$
\lim _{\delta \rightarrow 0} \tau(f, \delta)=0
$$

(все это равносильно интегрируемости по Риману).

Возможность сколь угодно точного одностороннего приближения класса конечными множествами означает компактность в некотором смысле. Поскольку одностороннее приближение соответствует покрытию множествами

$$
[P, Q]=\{f: P(x) \leqslant f(x) \leqslant Q(x) \text { для всех } x \in \mathbb{T}\},
$$

называемыми в англоязычной литературе "скобками" (brackets), будем употреблять термин "скобочная компактность", или “[]-компактность". Дадим формальное определение.

Пусть $\mathscr{F}$ - некоторый класс измеримых функций $f: \mathbb{T} \rightarrow \mathbb{R}$, для которых конечен интеграл $\|f\|_{1}=\int_{\mathbb{T}}|f(x)| d x$. Класс $\mathscr{F}$ назовем []-компактным, если для любого $\varepsilon>0$ существуют $N \in \mathbb{N}$ и функции $P_{i}, Q_{i}, i=1,2, \ldots, N$, такие, что

$$
\mathscr{F} \subset \bigcup_{i=1}^{N}\left[P_{i}, Q_{i}\right] \quad \text { и } \quad\left\|P_{i}-Q_{i}\right\|_{1}<\varepsilon, \quad i=1,2, \ldots, N .
$$

2. Связь $\tau$ и скобочной компактности. Мы установим критерий []-компактности в случае, когда $\mathscr{F}$ инвариантен относительно сдвигов, т.е. из $f(\cdot) \in \mathscr{F}$ и $h \in \mathbb{T}$ следует $f(\cdot-h) \in \mathscr{F}$.

Работа выполнена при поддержке Российского фонда фундаментальных исследований (гранты №№ ОФИ-М 09-01-12173 и 08-01-0028), и Программы "Ведущие научные школы" (грант № НШ-3810.2008.1). 


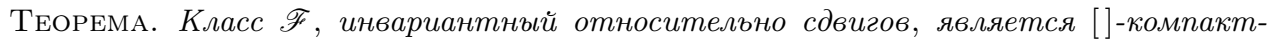
нъц, если и только если выполнены следующие условия:

- $\mathscr{F}$ равномерно ограничен: $\sup _{f \in \mathscr{F}, x \in \mathbb{T}}|f(x)|<\infty$;

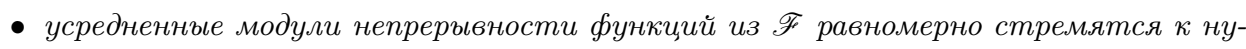
лю:

$$
\lim _{\delta \rightarrow 0} \sup _{f \in \mathscr{F}} \tau(f, \delta)=0
$$

ДокАЗАтЕльство. Пусть $\mathscr{F}$ []-компактен. Установим равномерную ограниченность. Пусть для какого-либо $\varepsilon>0$ класс $\mathscr{F}$ покрывается $N$ множествами $\left[P_{i}, Q_{i}\right]$. Покажем, чTо

$$
|f| \leqslant N \max _{i=1, \ldots, N} \max \left(\left\|P_{i}\right\|_{1},\left\|Q_{i}\right\|_{1}\right)
$$

Предположим, что $f\left(x_{0}\right)>C$. В силу инвариантности $\mathscr{F}$ относительно сдвигов существуют множества $H_{1}, \ldots, H_{N} \subset \mathbb{T}$ такие, что

$$
f(\cdot-h) \in\left[P_{i}, Q_{i}\right] \quad \text { для } h \in H_{i} \quad \text { и } \quad \bigcup_{i=1}^{N} H_{i}=\mathbb{T} .
$$

Найдется $i_{0}$ такой, что $\mu^{*}\left(H_{i_{0}}\right) \geqslant 1 / N$. Очевидно, $Q_{i_{0}}>C$ на множестве $x_{0}+H_{i_{0}}$, откуда $\int_{\mathbb{T}}\left|Q_{i_{0}}\right| d x \geqslant C / N$, т.е.

$$
C \leqslant N\left\|Q_{i_{0}}\right\|_{1} .
$$

Аналогично значения $f$ оцениваются снизу через $\left\|P_{i}\right\|_{1}$.

Установим равномерное стремление к нулю $\tau(f, \delta)$. Предположим, оно не имеет места: существуют $\varepsilon_{0}>0, \delta_{n}>0, f_{n} \in \mathscr{F}, n=1,2, \ldots$ такие, что $\delta_{n} \rightarrow 0$ при $n \rightarrow \infty$, но $\tau\left(f_{n}, \delta_{n}\right)>\varepsilon_{0}$. В силу равномерной ограниченности мы можем считать, что $|f| \leqslant 1$ для $f \in \mathscr{F}$. Предположим, для нашего класса выполнено (1) с $\varepsilon=\varepsilon_{0}^{3} / 130$ и некоторым $N<\infty$. Выведем отсюда противоречие.

Неравенства $|f| \leqslant 1$ и $\tau(f, \delta)>\varepsilon_{0}$ влекут

$$
\mu\left\{x \in \mathbb{T}: \exists x^{\prime}, x^{\prime \prime} \in\left(x-\frac{\delta}{2}, x+\frac{\delta}{2}\right) f\left(x^{\prime \prime}\right)-f\left(x^{\prime}\right)>\frac{\varepsilon_{0}}{2}\right\}>\frac{\varepsilon_{0}}{4} .
$$

Если $y^{\prime \prime}-y^{\prime}>t$, то $y^{\prime} \leqslant k \cdot t / 2<(k+1) \cdot t / 2 \leqslant y^{\prime \prime}$ для некоторого целого $k$. В нашем случае $y^{\prime}=f\left(x^{\prime}\right), y^{\prime \prime}=f\left(x^{\prime \prime}\right), t=\varepsilon_{0} / 2$, поэтому $y^{\prime}, y^{\prime \prime} \in[-1,1]$ и $k$ может принимать не более $8 / \varepsilon_{0}$ значений. Получаем

$$
\mu\left\{x: \exists x^{\prime}, x^{\prime \prime} \in\left(x-\frac{\delta}{2}, x+\frac{\delta}{2}\right) \quad f\left(x^{\prime}\right) \leqslant \frac{k \varepsilon_{0}}{4}<\frac{(k+1) \varepsilon_{0}}{4} \leqslant f\left(x^{\prime \prime}\right)\right\}>\frac{\varepsilon_{0}^{2}}{32}
$$

для некоторого $k \in \mathbb{Z} \cap\left[-4 / \varepsilon_{0}, 4 / \varepsilon_{0}-1\right]$.

Применяя рассуждения из предыдущего абзаца к $f_{n}$, получим, что для подходящих $k_{n}$ выполнены неравенства (2) с $f=f_{n}$. Переходя в последовательности $\left\{f_{n}\right\}$ к подпоследовательности, можно считать, что индекс $k_{n}$ у всех одинаковый: $k_{n}=k_{0}, n=1,2, \ldots$. Обозначим $\alpha=k_{0} \cdot \varepsilon_{0} / 4, \beta=\left(k_{0}+1\right) \cdot \varepsilon_{0} / 4$. Итак, мы получили, что множества

$$
A_{n}=\left\{x: \exists x^{\prime}, x^{\prime \prime} \in\left(x-\frac{\delta_{n}}{2}, x+\frac{\delta_{n}}{2}\right) \quad f_{n}\left(x^{\prime}\right) \leqslant \alpha<\beta \leqslant f_{n}\left(x^{\prime \prime}\right)\right\}
$$

имеют меру $\mu\left(A_{n}\right)>\varepsilon_{0}^{2} / 32$.

Воспользуемся инвариантностью относительно сдвигов:

$$
f_{n}(\cdot-h) \in\left[P_{i}, Q_{i}\right] \quad \text { при } \quad i \in H_{i}^{(n)} \quad \text { и } \quad \bigcup_{i=1}^{N} H_{i}^{(n)}=\mathbb{T} \quad \text { для всех } \quad n .
$$


Выберем индекс $i=i(n)$ такой, что $\mu^{*}\left(H_{i}^{(n)} \cap\left[0, \delta_{n}\right]\right) \geqslant \delta_{n} / N$. Переходя к подпоследовательности, можно считать, что индекс $i$ для всех $n$ один и тот же: $i(n)=i_{0}$.

Поскольку $\mu\left(A_{n}\right) \geqslant \varepsilon_{0}^{2} / 32$, то же неравенство верно для меры множества

$$
A=\bigcap_{n=1}^{\infty} \bigcup_{k=n}^{\infty} A_{k}
$$

Мы покажем, что $P_{i_{0}}(x) \leqslant \alpha<\beta \leqslant Q_{i_{0}}(x)$ для п.в. $x \in A$, откуда

$$
\left\|P_{i_{0}}-Q_{i_{0}}\right\|_{1} \geqslant(\beta-\alpha) \cdot \frac{\varepsilon_{0}^{2}}{32}=\frac{\varepsilon_{0}^{3}}{128},
$$

что даст противоречие.

Возьмем $B=\{x: P(x) \leqslant \alpha\}$. Если $\mu(A \backslash B)>0$, то найдется точка плотности $x_{0} \in$ $A \backslash B$; для нее существует $\gamma_{0}>0$ такое, что

$$
\mu\left(\left(x_{0}-\frac{\gamma}{2}, x_{0}+\frac{\gamma}{2}\right) \cap(A \backslash B)\right)>\gamma\left(1-\frac{1}{4 N}\right) \quad \text { для всех } \quad \gamma \in\left(0, \gamma_{0}\right) .
$$

По условию $\delta_{n} \rightarrow 0$; значит, $\delta_{n}<\gamma_{0} / 3$ при $n>n_{0}$. Так как $x_{0} \in A$, то $x_{0} \in A_{n}$ для некоторого $n>n_{0}$. Значит, $f_{n}\left(x_{1}\right) \leqslant \alpha$ для некоторого $x_{1} \in\left(x_{0}-\delta_{n} / 2, x_{0}+\delta_{n} / 2\right)$, откуда

$$
P_{i_{0}}(x) \leqslant \alpha \quad \text { для } \quad x \in x_{1}+H_{i_{0}}^{(n)} .
$$

Обозначим $\widetilde{H}=x_{1}+\left(H_{i_{0}}^{(n)} \cap\left[0, \delta_{n}\right]\right)$ и возьмем $\gamma=3 \delta_{n}$. Имеем $\widetilde{H} \subset\left(x_{0}-\gamma / 2, x_{0}+\gamma / 2\right)$, $\widetilde{H} \subset B$ и $\mu^{*}(\widetilde{H}) \geqslant \delta_{n} / N=\gamma \cdot(1 /(3 N))$ в силу выбора $i_{0}$. Но это противоречит (3). Значит, $P(x) \leqslant \alpha$ для п.в. $x \in A$; аналогично доказывается неравенство $Q(x) \geqslant \beta$. Необходимость условий доказана.

Установим []-компактность равномерно ограниченного класса с равномерно стремящимися к нулю усредненными модулями непрерывности (инвариантность можно не требовать). Рассмотрим множество $K_{n}$ функций, постоянных на полуинтервалах $[k / n$, $(k+1) / n)$. Погрешность одностороннего приближения

$$
E_{[]}\left(\mathscr{F}, K_{n}\right):=\sup _{f \in \mathscr{F}} E_{[]}\left(f, K_{n}\right)
$$

стремится к нулю при $n \rightarrow \infty$. Класс $K_{n} \cap\{f:|f| \leqslant C\}$ является, как легко проверить, []-компактным. Из этих двух утверждений следует []-компактность $\mathscr{F}$.

3. Равномерный закон больших чисел. В теории вероятностей []-компактность используется как достаточное условие сходимости эмпирических процессов, в частности, равномерного закона больших чисел (РЗБЧ).

Пусть $X_{1}, \ldots, X_{n}$ - независимые одинаково распределенные случайные величины; пусть $\mathscr{F}$ - некоторый класс функций $f$ с $\mathrm{E}\left|f\left(X_{1}\right)\right|<\infty$. В силу закона больших чисел для каждой $f \in \mathscr{F}$

$$
\lim _{n \rightarrow \infty}\left|\frac{1}{n} \sum_{i=1}^{n} f\left(X_{i}\right)-\mathrm{E} f\left(X_{1}\right)\right| \rightarrow 0 \quad \text { п.в., }
$$

РЗБЧ означает, что сходимость равномерна по $f \in \mathscr{F}$ :

$$
\lim _{n \rightarrow \infty} \sup _{f \in \mathscr{F}}\left|\frac{1}{n} \sum_{i=1}^{n} f\left(X_{i}\right)-\mathrm{E} f\left(X_{1}\right)\right|=0 \text { п.в. }
$$

Обозначим через $\mu$ распределение $X_{i}, \mu(A):=\mathrm{P}\left(X_{1} \in A\right)$. Хорошо известно (см., например, [2; гл. 2.4]) следующее 
УтвеРЖДЕНИЕ. Если $\mathscr{F}$ []-компактен в $L_{1}(\mu)$, то для него выполнен РЗБЧ (4).

Обычная компактность в $L_{1}$, конечно, не достаточна для РЗБЧ. С другой стороны, []-компактность не является необходимой. Класс характеристических функций последовательности независимых множеств $A_{n}$ меры $\mu\left(A_{n}\right)=1 / n$ удовлетворяет условию (4), но не является []-компактным (пример взят из [3; гл. 7.1]). Мы приведем в определенном смысле более сильный пример.

Везде далее будем говорить о РЗБЧ для класса множеств, имея в виду класс соответствующих характеристических функций.

Первыми вопрос о выполнении РЗБЧ для произвольного семейства множеств рассмотрели Вапник и Червоненкис [4]. Ими было получено достаточное условие для РЗБЧ в комбинаторных терминах. Именно, если $\mathscr{A}-$ семейство множеств, и для некоторого $d \in \mathbb{N}$ выполнено условие

$$
\forall B \quad|B|>d \quad \Longrightarrow \quad\left(\exists B^{\prime} \subset B \quad \forall A \in \mathscr{A} \quad A \cap B \neq B^{\prime}\right)
$$

$(|B|-$ мощность множества $B)$, то для $\mathscr{A}$ имеет место РЗБЧ (при любом распределении $\left.X_{i}\right)$. Максимальное такое $d$ называют VC-размерностью $\mathscr{A}$.

Пример. Пусть $\mathscr{A}$ - семейство сдвигов на $\mathbb{T}$ множества $[0,1) \cap \mathbb{Q}$. Тогда $\mathscr{A}$ имеет $\mathrm{VC}$-размерность 1, но не является []-компактным по лебеговой мере.

Условие на VC-размерность легко проверяется. Некомпактность сразу следует из теоремы, поскольку модуль $\tau$ везде равен 1.

\section{СПИСОК ЦИТИРОВАННОЙ ЛИТЕРАТУРЫ}

[1] Б. Сендов, В. Попов, Усредненные модули гладкости, Мир, М., 1988. [2] А. W. van der Vaart, J. A. Wellner, Weak Convergence and Empirical Processes, Springer Ser. Statist., Springer-Verlag, New York, NY, 1996. [3] R. M. Dudley, Uniform Central Limit Theorems, Cambridge Stud. Adv. Math., 63, Cambridge Univ. Press, Cambridge, 1999. [4] B. Н. Вaпник, А. Я. Червоненкис, ТВП, 16:2 (1971), 264-279. 